\title{
Parametric Study of Pile Response to Side-by-Side Twin Tunneling in Stiff Clay
}

\author{
Naeem Mangi \\ Department of Civil Engineering \\ Quaid-e-Awam University of \\ Engineering, Science \& Technology \\ Nawabshah, Pakistan \\ naeem08ce30@gmail.com
}

\author{
Daddan Khan Bangwar \\ Department of Civil Engineering \\ Quaid-e-Awam University of \\ Engineering, Science \& Technology \\ Nawabshah, Pakistan \\ daddan@quest.edu.pk
}

\author{
Hemu Karira \\ Department of Civil Engineering \\ Mehran University of Engineering \& \\ Technology \\ Khairpur Mir's, Sindh, Pakistan \\ engr.hemu07civil@gmail.com
}

\author{
Samiullah Kalhoro \\ Department of Civil Engineering \\ Quaid-e-Awam University of Engineering, Science \& Technology \\ Nawabshah, Pakistan \\ samiullahkalhoro63@gmail.com
}

\author{
Ghulam Rasool Siddiqui \\ Department of Civil Engineering \\ Mehran University of Engineering \& Technology \\ Khairpur Mir's, Sindh, Pakistan \\ ghulamrasoolsiddiqui@gmail.com
}

\begin{abstract}
A three dimensional coupled-consolidation numerical parametric study was carried out in order to gain new insight of single pile response to side-by-side twin tunneling in saturated stiff clay. An advanced hypo plasticity (clay) constitutive model with small-strain stiffness was adopted. The effects of relative to the pile tunnel depths were investigated by simulating the twin tunnels near the pile at various depths of tunnels, namely near the pile shaft, adjacent to the pile toe, and below the pile toe. It was found that the second tunneling in each case resulted in a larger settlement than the one due to the first tunneling with a maximum percentage difference of $175 \%$ in the case of twin tunneling near the mid-depth of the shaft. This occurred due to the degradation of clay stiffness around the pile during the first tunneling. Conversely, the first tunneling-induced bending moment was reduced substantially during the second tunneling. The most critical location of twin tunnels relative to the pile was found to be below the pile toe.
\end{abstract}

\section{Keywords-twin tunneling; pile foundation; parameteric study}

\section{INTRODUCTION}

Tunnel excavation-induced stress relief and ground movements inevitably cause additional deformation and stress on adjacent pile foundations. It is a major concern for designers and engineers to evaluate the adverse effects on the existing piles. To understand the pile-soil-tunnel interaction mechanism, many field monitoring studies and centrifuge model tests [1-3] and analytical solutions and numerical modeling studies [4-7] have been conducted. They all concluded that tunneling adjacent to existing pile foundations caused pile settlement, additional axial load on piles, and induced bending moments along the piles, which is unfavourable for piled foundations. Their magnitudes are likely depended on the relative locations of tunnels and piles. However, most previous studies have focused on the effects of a single tunnel on single piles and pile groups. In fact, twin tunneling is particularly favoured when developing underground transportation systems [8]. To obtain a satisfactory numerical model of the single pile response to sideby-side twin-tunneling, the analysis needs to take account of the soil's small strain non-linearity. In view of the aforementioned issues, this study aims to systematically investigate the settlement and load transfer mechanism of an existing single pile due to side-by-side twin tunnels in saturated stiff clay. To achieve these objectives, a three-dimensional coupled-consolidation numerical parametric study was carried out by varying the twin tunnel depths relative to the pile.

\section{THREE DIMENSIONAL COUPLED CONSOLIDATION ANALYSIS}

To investigate single pile response to side-by-side twin tunneling in stiff saturated clay, a three-dimensional coupled consolidation numerical parametric study was conducted. To facilitate validation of the numerical model, the tunnel diameter, pile diameter, embedded length, and clear distance between the pile and the tunnel were identical (in prototype scale) to those in the centrifuge test [9]. Figure 1 shows the elevation view of the configuration of a typical numerical simulation in which twin tunnels were excavated adjacent to the pile toe. The diameter of each tunnel $(D)$ was $6 \mathrm{~m}$. The embedded length $\left(L_{p}\right)$ and diameter $\left(d_{p}\right)$ of the pile were $18 \mathrm{~m}$ and $0.8 \mathrm{~m}$ respectively. The modeled pile represents a cylindrical reinforced concrete (grade 40, reinforcement ratio $=1$ ) with a bending moment capacity of $800 \mathrm{kNm}$. The center-to-center distance between each tunnel and the pile was $5.5 \mathrm{~m}(0.92 D)$. It is worth noting that, in reality, high-rise buildings are unlikely to be built on a single pile. This hypothesized study is a more virtual case [3]. This simplification was made to understand the settlement and load transfer mechanism more clearly. The length of each model tunnel (along its longitudinal direction) is $72 \mathrm{~m}$, which is 
equivalent to $12 D$. Each tunnel excavation was simulated in 28 steps. At each step, the tunnel advanced a distance of $2.5 \mathrm{~m}$ $(0.42 D)$ [2]. The time increment of one day for each step was adopted in the finite element analysis. A monitoring section was selected at the transverse centreline of the pile (i.e. $y / D=0$ ) as a reference for the tunnel advancements. The parametric study consisted of nine different numerical simulations (in total) in which the existing single pile was located between the twin tunnels, which were excavated one after the other on either side of the pile at various depths of tunnels $\left(z_{t}\right)$ relative to the pile length $\left(L_{p}\right)$, namely near the pile shaft $\left(z_{t} / L_{p}=0.67\right.$ and $0.83)$, adjacent to the pile toe $\left(z_{t} / L_{p}=1.00\right)$ and below the pile toe $\left(z_{t} / L_{p}=1.17,1.33,1.50,1.67,1.83\right.$ and 2.00$)$. In addition to these simulations, a pile load test $(L)$ was conducted numerically in "greenfield" conditions (i.e. with no tunnels present) to obtain the ultimate capacity of the pile in stiff clay. Based on this, the working load was then calculated with a factor of safety of 3.0. The obtained working load was applied to the pile in the parametric analysis simulating twin tunneling. Table I summarises the conducted numerical simulations.

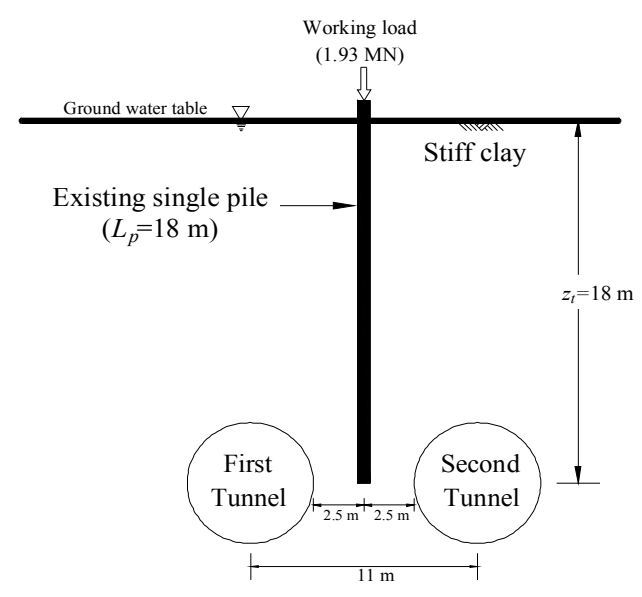

Note:- $L_{p}$ is embedded length of pile

$z_{t}$ is depth of tunnel axis from the ground surface

Fig. 1. Configuration of a typical numerical run representing case of $z_{t} / L_{p}=1.00$

TABLE I. NUMERICAL SIMULATIONS SUMMARY

\begin{tabular}{|c|c|c|}
\hline Description of numerical run & $\boldsymbol{z}_{\boldsymbol{t}} / \boldsymbol{L}_{\boldsymbol{p}}$ & $\boldsymbol{C} / \boldsymbol{D}$ \\
\hline \multirow{2}{*}{ Twin tunneling near the pile shaft } & 0.67 & $1.5,1.5$ \\
\cline { 2 - 3 } & 0.83 & $2.0,2.0$ \\
\hline Twin tunneling next to the pile toe & 1.00 & $2.5,2.5$ \\
\hline \multirow{4}{*}{ Twin tunneling below the pile toe } & 1.17 & $3.0,3.0$ \\
\cline { 2 - 3 } & 1.33 & $3.5,3.5$ \\
\cline { 2 - 3 } & 1.50 & $4.0,4.0$ \\
\cline { 2 - 3 } & 1.67 & $4.5,4.5$ \\
\cline { 2 - 3 } & 1.83 & $5.0,5.0$ \\
\cline { 2 - 3 } & 2.00 & $5.5,5.5$ \\
\hline
\end{tabular}

$z_{t}=$ tunnel depth, $L_{p}=$ pile length, $C / D=$ cover to diameter of tunnel ratio

\section{FINITE ELEMENT MESH AND BOUNDARY CONDTIONS}

Figure 2 shows an isometric view of a typical finite element mesh (for the case of $z_{t} / L_{p}=1.0$ ). The size of the mesh for each numerical simulation is $72 \mathrm{~m} \times 72 \mathrm{~m} \times 60 \mathrm{~m}$. These dimensions were sufficiently large to minimize the boundary effects in the numerical simulation because a further increase in the dimensions of the finite element mesh did not lead to any change in the computed results. Regarding the element size in the mesh, it is found that further halving the adopted mesh size leads to a change in the computed results of no more than only $0.2 \%$, suggesting that the mesh is sufficiently fine. Eight-noded hexahedral brick elements were used to model the soil and the pile. Four-noded shell elements were adopted to model each tunnel lining. Roller and pin supports were applied to the vertical sides and the base of the mesh, respectively. Therefore, movements normal to the vertical boundaries and in all directions of the base were restrained. The water table was assumed to be at the ground surface. Initially, the pore water pressure distribution was assumed to be hydrostatic. Free drainage was allowed at the top boundary of the mesh. The tunnel lining was assumed to be continuous and impervious.

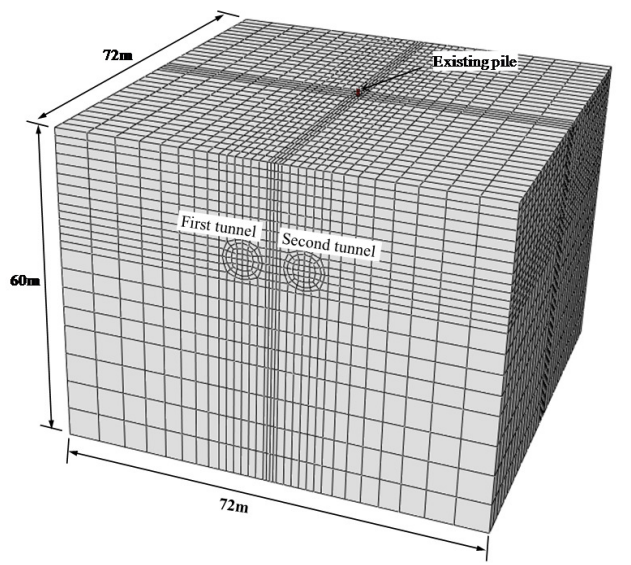

Fig. 2. Finite element mesh and boundary conditions of a typical numerical analysis $\left(z_{t} / L_{p}=1.00\right)$

Interaction between the piles and surrounding soil was modeled by the surface-to-surface contact provided in Abaqus software package [10]. The surface-to-surface contact considers the shape of both the master and slave surface in the contacting region and allows pile-soil friction. The penalty approach was used for tangential contact and the normal behavior was modeled as hard contact with no normal relative displacement between the pile and the surrounding soil. The interface was modelled by the Coulomb's friction law, in which the interface friction coefficient $(\mu)$ and limiting displacement $\left(\gamma_{\text {lim }}\right)$ are required as input parameters. A limiting shear displacement of $5 \mathrm{~mm}$ was assumed to achieve full mobilization of the interface friction equal to $\mu \times p^{\prime}$, where $p^{\prime}$ is the normal effective stress between two contact surfaces, and a typical value of $\mu$ for a bored pile of 0.35 was used [11]. The tunneling stress release process was modelled by the "element death" technique which is widely used in finite element analysis. In this technique, elements and nodes can be deactivated and activated. In this study, the volume loss is predefined before tunneling by specifying the area of the annulus gap between the tunnel lining and the excavated soil. Pattern of the non-uniform displacement boundaries was determined according to the displacement controlled model (DCM) in [5]. The soil inside the segment is excavated by deactivating soil elements inside it 
and by specifying zero horizontal displacement at the tunnel face of the tunnel segment to be excavated. In the meantime, the shell elements representing the tunnel lining are activated.

\section{CONSTITUTIVE MODEL AND MODEL PARAMETERS USED IN FINITE ELEMENT ANALYSIS}

A basic hypoplastic model was developed to capture the nonlinear behavior (upon monotonic loading at medium-to large-strain levels) of granular materials [12]. The basic model consists of five parameters (Table II). To account for the strain dependency and path dependency of the soil stiffness (at small strains), authors in [12] further improved the basic hypoplastic model by incorporating the concept of intergranular strain, which requires five additional parameters. The hypoplastic clay model with small strain stiffness has been implemented in the finite element software package Abaqus through a user-defined subroutine. The coefficient of lateral earth pressure at rest, Ko was estimated by the relevant equation in [14]. The concrete pile and tunnel lining were assumed to be linear elastic with Young's modulus of $35 \mathrm{GPa}$ and Poisson's ratio of 0.25 . The thickness of the lining was taken as $0.25 \mathrm{~m}$. The unit weight of concrete was taken as $24 \mathrm{kN} / \mathrm{m}^{3}$. The parameters for the piles and the tunnel lining are summarized in Table III.

TABLE II. ADOPTED MODEL PARAMETERS OF KAOLIN CLAY

\begin{tabular}{|l|c|}
\hline \multicolumn{1}{|c|}{ Description } & Value \\
\hline Effective angle of shearing resistance at critical state, $\phi^{\prime}$ & $22^{\circ}$ \\
\hline $\begin{array}{l}\text { Parameter controlling the slope of the isotropic normal } \\
\text { compression line in the } \ln (1+\mathrm{e}) \text { versus lnp plane, } \lambda^{*}[13]\end{array}$ & 0.11 \\
\hline $\begin{array}{l}\text { Parameter controlling the slope of the isotropic normal } \\
\text { compression line in the ln(1+e) versus lnp plane, } \kappa^{*}[13]\end{array}$ & 0.026 \\
\hline $\begin{array}{l}\text { Parameter controlling the position of the isotropic normal } \\
\text { compression line in the ln(1+e)-lnp plane, } N\end{array}$ & 1.36 \\
\hline $\begin{array}{l}\text { Parameter controlling the shear stiffness at medium- to } \\
\text { large- strain levels, } r\end{array}$ & 0.65 \\
\hline $\begin{array}{l}\text { Parameter controlling the initial shear modulus upon } 180^{\circ} \\
\text { strain path reversal, } m_{R}\end{array}$ & 14 \\
\hline $\begin{array}{l}\text { Parameter controlling the initial shear modulus upon } 90^{\circ} \\
\text { strain path reversal, } m_{T}\end{array}$ & 11 \\
\hline Size of elastic range, $R$ & $1 \times 10^{-5}$ \\
\hline $\begin{array}{l}\text { Parameter controlling the rate of degradation of the stiffness } \\
\text { with strain, } \beta r\end{array}$ & 0.1 \\
\hline $\begin{array}{l}\text { Parameter controlling the degradation rate of stiffness with } \\
\text { strain, } \chi\end{array}$ & 0.7 \\
\hline Initial void ratio, $e$ & 1.05 \\
\hline Dry density $\left(\mathrm{kg} / \mathrm{m}^{3}\right)$ & $1 \times 10^{-9}$ \\
\hline Coefficient of permeability, $k(\mathrm{~m} / \mathrm{s})$ & \\
\hline
\end{tabular}

TABLE III. ADOPTED CONCRETE PARAMETERS IN FEM

\begin{tabular}{|c|c|}
\hline Description & Value \\
\hline Young's Modulus, $\boldsymbol{E}$ & $35 \mathrm{GPa}$ \\
\hline Poisson's ratio, $\boldsymbol{v}$ & 0.3 \\
\hline Density, $\boldsymbol{\rho}$ & $2400 \mathrm{~kg} / \mathrm{m}^{3}$ \\
\hline
\end{tabular}

\section{INTERPRETATION OF COMPUTED RESULTS}

\section{A. Induced Pile Settlement Due to Twin Tunnels}

Figure 3 illustrates the pile settlement induced after the first and th second tunnel at different depths relative to the pile $\left(z_{t} / L_{p}\right)$. The long-term pile settlement (i.e. 15 years after twin tunneling completion) is included in the figure. The measured induced pile settlement tunneling at different depths in centrifuge modeling reported by $[9,15]$ is also shown in the figure for comparison. A linear increase in twin tunnelinginduced settlement was observed as the tunnel depth increased from $z_{t} / L_{p}=0.67$ to 1.33 . However, as the depth increased further $\left(1.50 \leq z_{t} / L_{p} \leq 2.0\right)$, the induced settlement decreased with a similar trend. This computed result is consistent with the one measured in centrifuge tests simulating tunneling at different depths relative to piles in stiff clay [9] and in sand [8]. This tunneling-induced settlement mechanism with $z_{t} / L_{p}$ can be attributed to the influence zone of tunneling-induced ground movement and stress-release-affected regions. In the case of $z_{t} / L_{p}=1.33$, the entire pile stood within the influence zone of tunneling-induced ground movement, and the stress release region was developed directly underneath the pile toe. When $z_{t} / L_{p}=0.67,0.83$ and 1.00 , the pile was located only partially within the influence zones. Therefore, the pile experienced larger settlement in the case of $z_{t} / L_{p}=1.33$ than in these cases. Although the entire pile in the cases of $z_{t} / L_{p}=1.50,1.67,1.83$ and 2.00 was located inside the tunneling-induced ground movement, the tunneling-induced pile settlement was less than that of $z_{t} / L_{p}=1.33$. This is because the location of the pile toe was beyond the tunneling-induced stress-release-affected region in these cases.

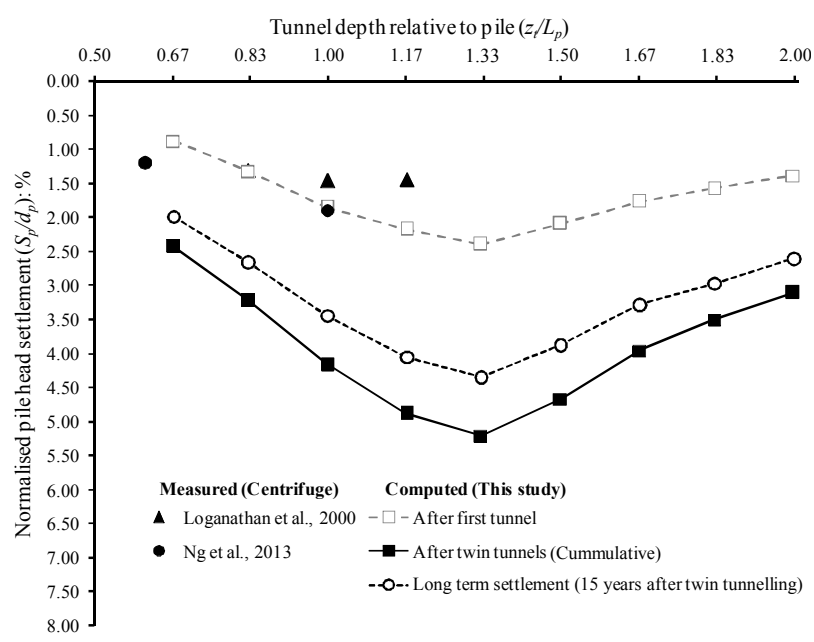

Fig. 3. Computed load settlement curve from the pile load test without tunneling

Qualitatively, the second tunneling-induced settlement trend with respect to $z_{t} / L_{p}$ was similar to the first tunnelinginduced settlement. However, the magnitude of the pile settlement induced by the second tunnel was higher than that induced by the first tunnel in each case. This can be attributed to the degradation of clay stiffness around the pile due to stress release and the development of shear strains as a result of the first tunnel excavation. The largest settlement $\left(175 \%\right.$ of $S_{p}$ due to the first tunnel) was induced by the second tunnel when $z_{l} / L_{p}=0.67$. It can be seen that the settlement of the pile reduced in the long term (i.e. 15 years after completion of the twin tunnels) in all cases. This pile heave is attributed to the dissipation of excessive negative pore pressure generated 
around the pile due to the twin tunnels. The maximum reduction of the pile settlement $(17.2 \%$ of the settlement after twin tunnels) was observed in the case $z_{t} / L_{p}=1.33$.

\section{B. Changes in Axial Load Distribution}

Figure 4 illustrates the axial force distribution along the pile with normalized depth (i.e., $Z / L_{p}$ ) below the ground surface after twin tunneling when $z_{t} / L_{p}=0.67,1.00$ and 1.33 . The axial load distribution before tunneling (after applying the working load) is also included in the Figure as a reference. Before tunneling, the pile carried approximately $75 \%$ of the working load (i.e. $1010 \mathrm{kN}$ ) with its shaft resistance and the remainder with its end-bearing resistance. Because tunneling was carried out near the mid-depth of the pile shaft in the case $z_{t} / L_{p}=0.67$, tunnel-induced reduction in normal stresses to the pile shaft and downward soil movement caused an increase in axial load along the entire length of the pile after the first and second tunnel excavations. At the end of the first tunneling, the maximum increment in the axial force $(63 \%$ of that at working load) was computed at $Z / L_{p}=0.7$, which is above the tunnel spring line. By inspecting the axial load distribution after the first tunnel excavation, it is observed that along the upper half of the pile $\left(0 \leq Z / L_{p} \leq 0.6\right)$, the shaft resistance decreases to zero. Consequently, the load was transferred to the lower half of the pile. To maintain equilibrium, the pile had to settle to further mobilize the end-bearing and shaft resistance along the lower portion $\left(Z / L_{p}>0.6\right)$. This led to increases of $73 \%$ and $24 \%$ in the mobilized end-bearing and shaft resistance at the lower portion $\left(Z / L_{p}>0.5\right)$ respectively upon completion of the first tunnel. The second tunneling in the case $z_{t} / L_{p}=0.67$ caused a further reduction of the normal stresses to the pile shaft. Consequently, soil settled more than the pile, resulting in Negative Skin Friction (NSF) along the upper half of the pile $\left(0 \leq Z / L_{p} \leq 0.6\right)$. This suggests that this portion of the pile is subjected to dragload by the surrounding soil. This caused the pile to settle more than that due to the first tunnel. To maintain vertical equilibrium of the pile, the soil surrounding the lower part of the pile $\left(Z / L_{p}>0.6\right)$ resisted its settlement by mobilizing Positive Skin Friction (PSF) at the pile-soil interface and endbearing resistance at the toe of the pile. Owing to the second tunneling, the end-bearing and mobilized shaft resistance increased to $190 \%$ and $18 \%$, respectively.

Owing to twin tunneling adjacent to the pile toe $\left(z_{t} / L_{p}=1.00\right)$, the axial load increased along the pile at depths ranging from 0.42 to $1.0 L_{p}$. The increase in axial load resulted from reduced shaft resistance, which was caused by stress release from twin tunneling adjacent to the pile toe. The reduction percentages in shaft resistance were $22 \%$ and $43 \%$ after the first and twin tunneling (cumulative) respectively. Consequently, the axial load borne by the shaft resistance along the pile at depths ranging from 0.42 to $1.0 L_{p}$ was transferred downward to the pile toe, leading to a $75 \%$ and $156 \%$ increase in mobilized end-bearing resistance after the first and twin (cumulative) tunneling, respectively. In contrast to the tunneling near the mid-depth of the pile shaft $\left(z_{t} / L_{p}=0.67\right)$ and adjacent to the pile toe $\left(z_{t} / L_{p}=1.00\right)$, the axial load decreased along the entire length of the pile when twin tunnels were excavated below the pile toe $\left(z_{t} / L_{p}=1.33\right)$. The advancement of the twin tunnels led to reductions in the end-bearing of the pile as a result of stress release from $2 \%$ volume loss. To compensate for the decrease in end-bearing resistance, the pile had to settle substantially to mobilize the shaft resistance along the entire pile length. This result is similar to those measured in the field [16]. The end-bearing decreased by $12 \%$ and $28 \%$ owing to the first and twin tunnels, respectively.

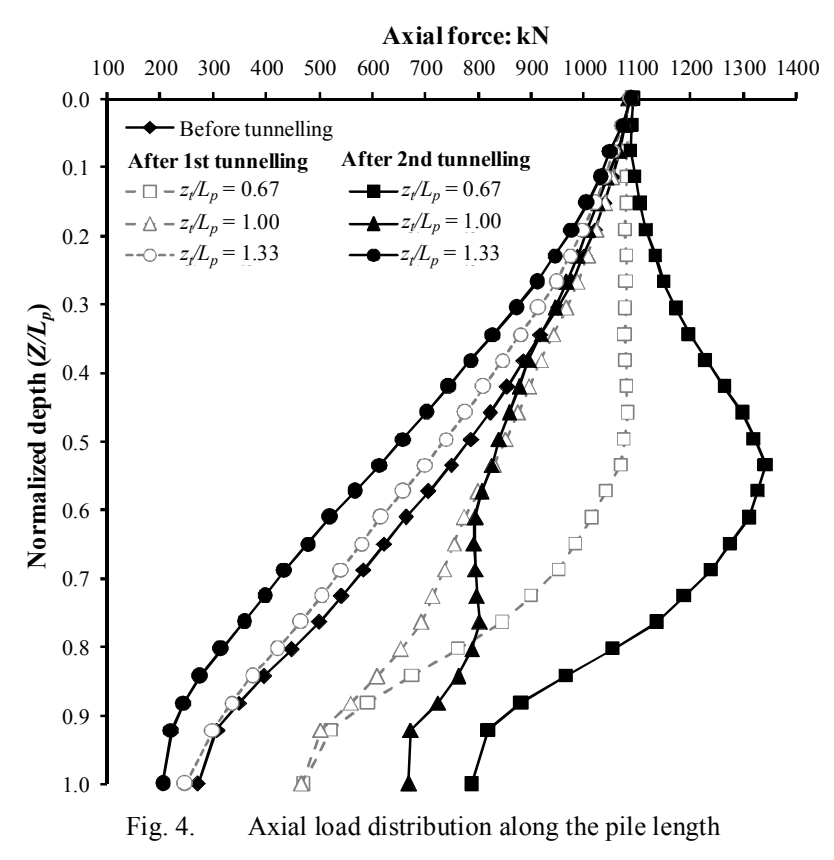

C. Twin-tunneling Induced Bending Moment along the Pile

Figure 5 illustrates the induced bending moment along the pile after the first and second tunneling in the cases of $z_{t} / L_{p}=0.67,1.00$ and 1.33. A positive bending moment means that tensile stress was induced along the pile shaft facing the first tunnel. The measured bending moment of a single pile subjected to single tunneling in centrifuge model test [9] was also included for comparison. Since there was no rigid constraint at the pile head (pile head was free to move and rotate), no bending moment was induced at/near the head of the pile in all the cases. It can be observed that in the case of $z_{t} / L_{p}=0.67$, the maximum positive bending moment was induced in the pile at $Z / L_{p}=0.6$ near spring line of the first tunnel. Authors in [17] have also reported a measured maximum bending moment occurring at the spring line of the tunnel near the shaft of the pile group. This happened because the pile was subjected to lateral soil movement towards the tunnel resulting from significant stress release. The magnitude of the maximum positive bending moment was $400 \mathrm{kNm}$ (which is $50 \%$ of the pile BM capacity). On the other hand negligible bending moment was induced near the pile toe (below the tunnel spring line), because the tunneling induced soil movement below the tunnel spring line was insignificant [2]. Subsequently, the pile was subjected to stress release on the opposite side of the pile as a result of the second tunnel's excavation near the mid-depth of the pile shaft. Consequently, the induced-bending moment decreased along the pile after the second tunneling. The induced-bending moment along the pile was not returned to zero after the second tunneling as the soil does not behave elastically. Finally, a positive bending moment 
with magnitude of $50 \mathrm{kNm}$ was induced. Owing to the soil inward movement towards the first tunnel when $z_{t} / L_{p}=1.00$, positive bending moment was induced at the lower part of the pile $\left(0.7 \leq Z / L_{p} \leq 1.0\right)$. To counter-balance this induced positive bending moment, negative bending moment was developed along the upper part of the pile $\left(0 \leq Z / L_{p} \leq 0.7\right)$. The magnitudes of maximum induced positive and negative bending moment were $130 \mathrm{kNm}$. The measured induced bending moment along the pile due to single tunneling in centrifuge reported by [9] was positive and smaller than the one computed in magnitude. This may be caused by the tunneling-induced volume loss which was modeled as $1 \%$ in the centrifuge test. The subsequent tunneling at $z_{t} / L_{p}=1.00$ on the opposite side of the pile reduced the induced bending moment significantly. The maximum induced bending moment was $37 \mathrm{kNm}$ at $Z / L_{p}=0.6$ after the twin tunnel excavation.

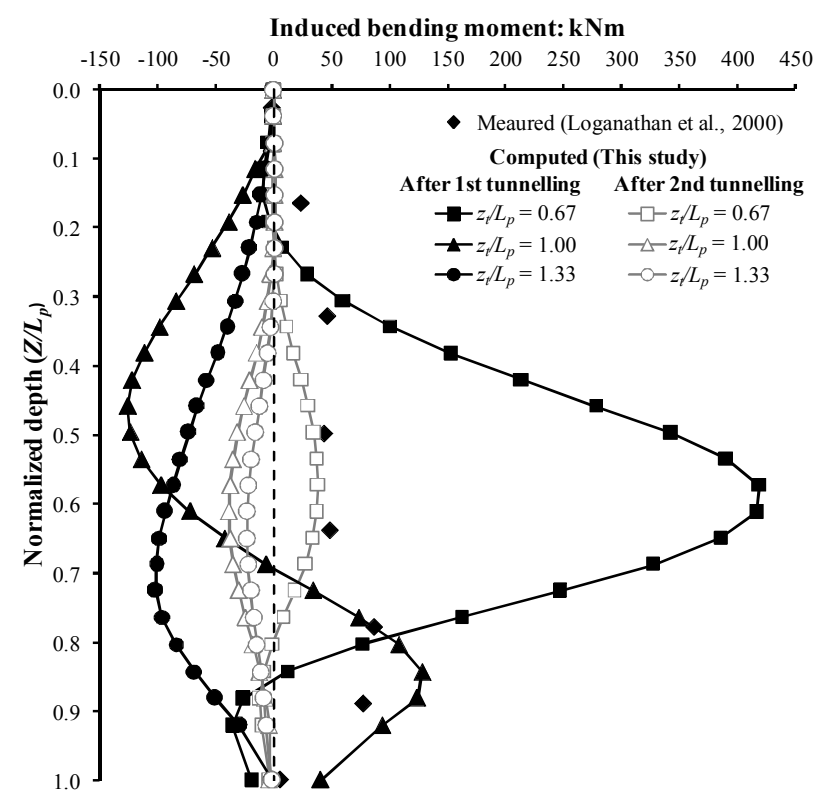

Fig. 5. Induced bending moment along the pile length

In contrast to the induced bending moment in the case $z_{\ell} / L_{p}=0.67$, negative bending moment was induced along the entire pile length due to the first tunnel advancement in the case of $z_{t} / L_{p}=1.33$. However, the magnitude of maximum bending $\left(100 \mathrm{kPa}\right.$ at $\left.Z / L_{p}=0.7\right)$ was less than the one in the case $z_{t} / L_{p}=0.67$. Also, no bending was induced at the pile toe and head. It can be seen that the induced bending moment during the first tunnel advancement in the case of tunneling near the mid-depth of the pile $\left(z_{t} / L_{p}=0.67\right)$ and adjacent the pile toe $\left(z_{t} / L_{p}=1.00\right)$ was less than that in the case of tunneling below the pile toe $\left(z_{t} / L_{p}=1.33\right)$. Therefore, in the case of $z_{t} / L_{p}=1.33$, the most critical issue to be considered is the relatively large settlement.

\section{Discussion}

It is well recognized that the stress-strain relationship of soils is highly nonlinear even at very small strains. The stiffness of most soils decreases as strain increases and depends on the recent stress or strain history of the soil. Owing to non- linear soil behavior, a tunnel excavation can cause reduction in the stiffness of the soil. Therefore, it is vital to investigate the pile responses not only to the first tunnel but also to the subsequent tunnel in clay in a twin-tunneling transportation system. Keeping these issues into consideration, effects of twin side by side tunneling on a single pile in stiff clay were investigated in this study. It was revealed that the second tunneling caused larger settlement and smaller bending moment in the pile. This finding is the major contribution of this parametric study.

\section{CONCLUSIONS}

Based on the modeled ground conditions, geometry, and tunneling method, the following conclusions can be drawn:

- Due to the degradation of the stiffness of the clay surrounding the pile as a result of tunneling-induced stress release and shear strain, the second tunneling caused larger settlement than the first in each case. When $z_{t} / L_{p}=0.67$ the second tunneling-induced settlement was the largest (i.e. $175 \%$ of $S_{p}$ from the first tunnel) of all cases. The cumulative settlements of the pile resulting from the working load and twin tunneling in the cases $z_{t} / L_{p}=0.67$, 1.00 and 1.33 were 25,39 , and $47 \mathrm{~mm}$ (i.e. $3.1,4.9$, and 5.9 of the pile diameter) respectively.

- The first tunneling in the case $z_{t} / L_{p}=0.67$ induced the largest bending moment (50\% of the pile BM capacity) at the spring line of the tunnel. However, the induced bending moment along the pile decreased significantly due to the excavation of the second tunnel in each case because of the side-by-side twin tunneling configuration in which the second tunnel caused a stress release on the opposite side of the pile.

- When of $z_{t} / L_{p}=0.67$, the second tunneling-induced soil movement due to stress release mobilized negative shaft friction at the upper part of the pile. Consequently, a downward load transfer was observed along the pile, further mobilizing the pile end-bearing. Similarly, in the case $z_{t} / L_{p}=1.00$, the load borne by the pile shaft was transferred to the pile toe as a result of the twin tunneling reduction in the shaft resistance at the lower part of the pile $\left(0.3 \leq Z / L_{p} \leq 0.9\right)$.

\section{ACKNOWLEDGEMENT}

The authors would like to acknowledge the financial support provided by the Quaid-e-Awam University of Engineering, Science \& Technology, Sindh and Pakistan.

\section{REFERENCES}

[1] G. Gudehus, "A comprehensive constitutive equation for granular materials", Soils and Foundations, Vol. 36, No. 1, pp. 1-12, 1996

[2] K. Ishihara, "Liquefaction and flow failure during earthquakes", Geotechnique, Vol. 43, No. 3, pp. 351-451, 1993

[3] C. W. W. Ng, "The state-of-the-art centrifuge modelling of geotechnical problems at HKUST", Journal of Zhejiang University Science A, Vol. 15 , pp. $1-21,2014$

[4] J. Jaky, "The coefficient of earth pressure at rest", Journal of the Society of Hungarian Architects and Engineers, pp. 355-358, 1944 
[5] K. H. Chiang, C. J. Lee, "Responses of single piles to tunneling-induced soil movements in sandy ground", Canadian Geotechnical Journal, Vol. 44, No. 10, pp. 1224-1241, 2007

[6] A. Niemunis, I. Herle, "Hypoplasic model for cohesionless soils with elastic strain range", Mechanics of Cohesive-frictional Materials, Vol. 2, No. 4, pp. 279-299, 1997

[7] C. W. W. Ng, M. A. Soomro, Y. Hong, "Three-dimensional centrifuge modelling of pile group responses to side-by-side twin tunnelling", Tunelling and Underground Space Technology, Vol. 43, pp. 350-361, 2014

[8] C. W. W. Ng, H. Lu, S. Y. Peng, "Three-dimensional centrifuge modelling of the effects of twin tunnelling on an existiong pile", Tunneling and Underground Space Technology, Vol. 35, pp. 189-199, 2013

[9] N. Loganathan, H. G. Poulos, D. P. Stewart, "Centrifuge model testing of tunnelling-induced ground and pile deformations", Geotechnique, Vol. 50, No. 3, pp. 283-294, 2000

[10] Abaqus user's manual, version 6.8.2, Dassault Systemes, 2008

[11] D. Masin, "Hypoplastic cam-clay model", Geotechnique, Vol. 62, No. 6, pp. 549-553, 2012

[12] A. M. Marshall, R. J. Mair, "Tunneling beneath driven or jacked endbearing piles in sand", Canadian Geotechnical Journal, Vol. 48, No. 12, pp. 1757-1771, 2011

[13] S. W. Jacobsz, J. R. Standing, R. J. Mair, T. Hagiwara, T. Sugiyama, "Centrifuge modelling of tunnelling near driven piles", Soils and Foundations, Vol. 44, No. 1, pp. 49-56, 2004

[14] P. W. Mayne, F. H. Kulhawy, "K0-OCR relationships in soils", Journal of the Geotechnical Engineering-ASCE, Vol. 108, No. 6, pp. 851-872, 1982

[15] D. A. Mangejo, M. A. Soomro, N. Mangi, I. A. Halepoto, I. A. Dahri, "A parametric study of effect on single pile integrity due to an adjacent excavation induced stress release in soft clay", Engineering, Technology \& Applied Science Research, Vol. 8, No. 4, pp. 3189-3193, 2018

[16] M. A. Soomro, A. S. Brohi, D. K. Bangwar, S. A. Bhatti, "3D numerical modelling of pile group responses to excavation-induced stress release in Silty Clay", Engineering, Technology \& Applied Science Research, Vol. 8, No. 1, pp. 2577-2584, 2018

[17] D. R. Coutts, J. Wang, "Monitoring of reinforced concrete piles under horizontal and vertical loads due to tunnelling", International Conference on Tunnels and Underground Structures, Singapore, November 26-29, 2000 